Description of the problem Involvement of community stakeholders in research often includes pre-grant solicitation of support or end-phase KT activities. To facilitate optimal and timely input from key stakeholders at all stages of research, the Alberta Program in Youth Sport and Recreational Injury Prevention developed a Knowledge Broker (KB) model.

Results (effects/changes) In alignment with research priorities, KBs were identified in relevant community partner organisations (Ever Active Schools, Hockey Calgary, WinSport). KBs bridge the gap between research, education and KT priorities within the academic institution and the community. KB activities include contributing to research questions, intervention development, subject recruitment, implementation and dissemination of findings. Linking researchers and knowledge users facilitates collaboration, a greater understanding of common and diverse goals, and new partnerships. The ideal outcome of these partnerships includes optimal knowledge exchange to maximise the uptake of research evidence for the greatest public health impact. A financial contribution from the research program helps support the commitment of KBs.

Conclusions The integrated nature of the research program with academic and community stakeholder partnerships, creates an ideal setting for enhancing the impact of $\mathrm{KT}$. KB involvement contributes significantly to achieving research objectives. KB contributions optimise the translation of research findings into injury prevention practice, programs and policies.

\section{SAFE COMMUNITY IN MALAYSIA: A WISH AND A DREAM}

${ }^{1,2} \underline{R}$ Annapoornam, ${ }^{3}$ S Kulanthayan. ' SJK Tamil Kajang Parents Volunteer Group; ${ }^{2}$ Universiti Putra Malaysia; ${ }^{3}$ Safe Kids Malaysia

10.1136/injuryprev-2016-042156.281

Background Safe Community has benefited many countries which adopted it for many years. Malaysia has been exploring this initiative since year 2012 under the Ministry of Housing and Local Government then. Universiti Putra Malaysia (UPM) is championing this initiative with a high hope to have a serious buying in from any interested community.

Methods The approach undertaken by UPM is by identifying possible community. We took both (top-down and also bottomup approach) approach in our attempt to win the hearts of the stakeholders. For the 'top-down' approach, we presented to two government authorities - Ministry of Housing and Local Government and the next one Putrajaya Corporation the Safe Community Concept. For the 'bottom-up' approach, we are working closely with a community in Kajang, Selangor.

Results The initial feedback are positive from this two local authorities for the 'top-down approach'. However we have not moved much and far as we detailed down the operational part. The 'bottom-up' approach also is giving us the initial positive sign as we erode into a segment of the community (school children, teachers, parents and organisation that supports children betterment). For the 'bottom-up' approach with the Kajang Community, UPM has embarked and facilitated few safety programs namely: 1) Medical Camps and Health Screening including Dental Services and Pharmacy, 2) Weekly Swim Safety Classes for Children, Parents and Teachers, 3) Pedestrian Safety Intervention for the entire community, 3) Eye Project to Improve Children Vision, 4) Fire and Electrical Safety Demonstration for a segment of the community, 5) Importance of Food Safety and Healthy Food for School Community, 6) Lab Safety for the community who deals with lab and finally 7) Crime Prevention talks and session with the Kajang Police Station Officials.

Conclusions In Safety 2016 Conference, we would be able to share more information on bringing in Safe Community to Malaysia and it is our wish to announce that we have the first community who is interested and applied to join the Safe Community movement.

\section{Child Maltreatment}

\section{Parallel Tue 2.2}

\section{CHILD PROTECTION AND DISABILITY IN SCOTLAND: A CAUSE FOR CONCERN}

${ }^{1}$ Julie Taylor, ${ }^{2}$ Christine Jones. ${ }^{1}$ University of Birmingham, UK; ${ }^{2}$ University of Strathclyde, UK

\subsection{6/injuryprev-2016-042156.282}

Background Disabled children are at least four times more likely to be abused than their non-disabled peers. This abuse is likely to be of longer duration and be more severe. Despite this heightened risk, the abuse of disabled children often goes undetected and underreported. This qualitative study investigated the specific issues faced by practitioners in Scotland in supporting disabled children at risk of significant harm.

Methods The aim of this study, commissioned by the Scottish Government, was to assess how public services (including social work, health care, education, police and other related services) identify and support disabled children at risk of significant harm, whether neglect or abuse.

This study focused on children and young people with a wide range of impairments, including physical, sensory, cognitive and communication impairments and those with mental distress, all of whom are disabled by external barriers. The study involved four concurrent components: telephone interviews, focus groups with Child Protection Committees (CPCs), practice case studies and the development of systems and response models. Interviews were held with participants from six local authority areas and across five different services. We also held five focus groups with Child Protection Committees (61 participants in total). Inductive analysis following framework design was undertaken.

Results There were positive messages about putting the child at the very heart of child protection assessment and intervention, regardless of any impairment a child may have. However, there was also concern that practice was at times parent-centred. Some participants appeared to be 'muddling through' in practice and many practitioners lacked confidence when working with disabled children. Data from this study suggests that thresholds for disabled children may be higher than for non-disabled children. Participants reported high levels of interagency working and saw this as inherently positive, although they recognised some failings and tensions.

Conclusions There is widespread commitment across the child protection system to putting the child at the centre. However, getting it right for every child does not mean treating every child the same. 


\section{3 IMPROVING US NATIONAL DATA ON CHILD MALTREATMENT FATALITIES}

${ }^{1}$ Patricia Schnitzer, ${ }^{2}$ Robert Anderson, ${ }^{2}$ Arialdi Minino, ${ }^{3}$ Theresa Covington. ${ }^{1}$ University of Missouri, USA; ${ }^{2}$ National Centre for Health Statistics, Centres for Disease Control and Prevention, USA; ${ }^{3}$ US National Centre on Fatality Reviews

\subsection{6/injuryprev-2016-042156.283}

Background In the United States (US), national data on child maltreatment $(\mathrm{CM})$ deaths are compiled from state child welfare agencies; approximaly $1600 \mathrm{CM}$ deaths are reported for all 50 states annually. Unfortunately, these child welfare data grossly undercount fatal CM in the US. This project used data from death certificates and child death review (CDR) programs to improve identification and better estimate fatal CM in the US.

Methods CDR data from 9 states with a high proportion of deaths reviewed and recorded in the National CDR Case Reporting System were linked to death certificate/mortality data submitted to the US National Centre for Health Statistics (NCHS). Case inclusion criteria were: children between 7 days and 5 years old that died during 2009 or 2010. A CM classification algorithm that included categories of presumptive, probable, possible CM was developed and applied to the linked deaths. Weights were calculated for each cause of death in each CM category; national CM mortality was estimated by applying these weights to 2011 NCHS mortality data for all 50 states.

Results A total of 10927 deaths meeting the inclusion criteria were identified. Of these, 6660 deaths linked to a record in the NCHS mortality file; 169 additional deaths identified by CDR did not link to a NCHS file record. The NCHS mortality file contained an additional 4098 deaths that did not have a CDR review. A total of 3606 deaths met the definition for presumptive $(\mathrm{n}=907)$, probable $(\mathrm{n}=387)$, or possible $(\mathrm{n}=2312) \mathrm{CM}$ in these 9 states over the 2 study years. This resulted in a national estimate of 1279 presumptive, 500 probable, and 3083 possible $\mathrm{CM}$ deaths for a total of $4862 \mathrm{CM}$ deaths in the US during 2011.

Conclusions It is possible to improve upon the current child welfare-based system of counting fatal child maltreatment in the US by linking CDR and mortality data. In this study, the CDR Program data were essential for confirming child maltreatment in many of the deaths identified.

\section{UNDERSTANDING THE IMPACT OF ADVERSE CHILDHOOD EXPERIENCES ON HEALTH AND WELLBEING IN ENGLAND}

${ }^{1}$ Kat Ford, ${ }^{1}$ Zara Quigg, ${ }^{1}$ Karen Hughes, ${ }^{1}$ Nadia Butler, ${ }^{1,2}$ Mark A Bellis. 'Liverpool John Moores University, United Kingdom; ${ }^{2}$ Public Health Wales, United Kingdom

\subsection{6/injuryprev-2016-042156.284}

Background An emergent body of research is underlining the long-term impacts that adverse childhood experiences (ACEs) have in determining the health and social prospects of individuals. ACEs are now considered one of the strongest predictors of poor health and social outcomes in adults. Individuals with higher numbers of ACEs are found to have higher risks of antisocial behaviour, crime, and violence, and are at increased risk of developing a range of health conditions and ultimately premature mortality.

Methods A household survey was conducted with 5,454 residents aged between 18 and 69 in the United Kingdom in the areas of Northamptonshire, Hertfordshire and Luton during the period June to September 2015. The survey explored childhood experiences before the age of 18, past and current health and social behaviours and outcomes.

Results Four in ten (43.1\%) of all individuals surveyed experienced at least one of the nine ACEs examined. The independent impact of ACEs on health and criminal justice outcomes will be discussed, including the increased risks of harmful behaviours, morbidity and mortality in adulthood from the number of ACEs. The associations between ACEs and socioeconomic markers will be identified. The presentation will provide an overview of the burden of harmful behaviours that would be prevented in the absence of ACEs. Findings across the study areas will be discussed in relation to prevalence of ACEs found in other research within the United Kingdom.

Conclusions The implications of the research findings on interventions to improve health and reduce inequalities will be highlighted. The importance of service planning and effective investment in early years at a local and national level to prevent ACEs and ensure the provision of positive childhood environments for future generations will be addressed.

\section{MARKOV MODEL SIMULATION OF PAEDIATRIC ABUSIVE HEAD TRAUMA PRIMARY PREVENTION AND SCREENING PROGRAMS}

1,2 Marion Bailhache, ${ }^{2,3}$ Antoine Bénard, ${ }^{2,3}$ Louis-Rachid Salmi. ' CHU De Bordeaux, Pole De Pediatrie, France; '2Université De Bordeaux, ISPED, Centre INSERM U897-EpidemiologieBiostatistique, France, ${ }^{3} \mathrm{CHU}$ De Bordeaux, Pole De Sante Publique, Service D'information Medicale, France

\subsection{6/injuryprev-2016-042156.285}

Background Paediatric Abusive Head Trauma (PAHT) is an important cause of morbidity and child-abuse deaths. Several prevention programs exist but their impact is difficult to estimate by lack of knowledge about the beginning of PAHT, difficulties to identify all cases, and the need for large cohorts. We modelled the potential impact of primary prevention and screening programs of PAHT in a hypothetical cohort of 800,000 newborns.

Methods We developed Markov models without intervention, and with primary prevention based on current interventions, screening during repeated consultations, addressed to all families until children are two-years old, and with both programs. Several scenarios were tested regarding potential side effects for parents being mislabeled as abusers (assumed or not to increase the risk of PAHT and decrease participation in screening) and length of the preclinical phase. The time horizon was two years with cycles of fifteen days. Outcomes were number of deaths and abused children avoided. Uncertainty was specified with probability distributions based on literature, or extreme possible values in absence of data.

Results After two years, the median number of death avoided through primary prevention would vary from 6 (CI: 95\%: 2-11) to $28(6-51)$ for 100000 newborns, depending on the probability of PAHT and scenario. Screening could prevent up to $6(0-29)$ deaths or cause up to $66(0-361)$ deaths for 100,000 children born alive. The impact of both programs on mortality and morbidity was uncertain.

Conclusions Our model confirmed the potential benefits of primary prevention and documented the huge uncertainty regarding benefits associated with screening of PAHT. Better estimating the effects of wrongly identifying caregivers as abusers, the evolution of PAHT, and the performance of tests to earlier identify childabuse are necessary. 